\title{
sciendo
}

\section{Characteristics of Functional Stability in Young Adolescent Female Artistic Gymnasts}

\author{
by \\ Agnieszka Opala-Berdzik1, Magdalena Głowacka², Kajetan J. Słomka
}

The aim of this study was to determine whether young adolescent female artistic gymnasts demonstrate better functional stability than age- and sex-matched non-athletes. Different characteristics of the gymnasts' postural control were expected to be observed. Twenty-two 10- to 13-year-old healthy females (ten national-level artistic gymnasts and twelve non-athletes) participated in the study. To assess their forward functional stability, the 30-s limit of stability test was performed on a force plate. The test consisted of three phases: quiet standing, transition to maximal forward leaning, and standing in the maximal forward leaning position. Between-group comparisons of the directional subcomponents of the root mean squares and mean velocities of the center of pressure and rambling-trembling displacements in two phases (quiet standing and standing in maximal leaning) were conducted. Moreover, anterior stability limits were compared. During standing in maximal forward leaning, there were no differences in the center of pressure and rambling measures between gymnasts and non-athletes $(p>0.05)$. The values of trembling measures in both anterior-posterior and medial-lateral directions were significantly lower in gymnasts $(p<0.05)$. Both groups presented similar values for anterior stability limits $(p>0.05)$. The comparisons of rambling components may suggest a similar supraspinal control of standing in the maximal leaning position between gymnasts and healthy non-athletes. However, decreased trembling in gymnasts may indicate reduced noise in their postural control system possibly due to superior control processes at the spinal level. The anterior stability limit was not influenced by gymnastics training in female adolescents.

Key words: postural stability, limits of stability, artistic gymnastics, female adolescents.

\section{Introduction}

In the postural control system, the central nervous system (CNS) regulates sensory information from the visual, vestibular, and somatosensory systems in order to produce adequate motor output to maintain a controlled body posture. Postural control concerns the inherent interrelation between the distribution of tonic muscle activity ("posture") and the adaptation to internal or external perturbations ("equilibrium") (Ivanenko and Gurfinkel, 2018). Proper postural stability enables performance of complex motor skills without losing balance. Although the mechanisms underlying human postural control have been widely investigated, there is still a great deal unknown about this issue. Expanding this knowledge may lead to the improvement of athletic training methods as well as physical therapy procedures for patients with postural control system dysfunctions.

It has been documented that functioning of the postural control system develops with age (Barela et al., 2003; Newell et al., 1997; Riach and Starkes, 1993; Usui et al., 1995) and it

\footnotetext{
1 - Institute of Physiotherapy and Health Sciences, Department of Physiotherapy in Internal Diseases, The Jerzy Kukuczka Academy of Physical Education, Katowice, Poland.

2 - Institute of Sport Sciences, Department of Individual Sports, The Jerzy Kukuczka Academy of Physical Education, Katowice, Poland.

3 - Institute of Sport Sciences, Department of Motor Behavior, The Jerzy Kukuczka Academy of Physical Education in Katowice, Poland.
} 
reaches maturity in the second decade of life (Ferronato and Barela, 2011; Figura et al., 1991; Goulème et al., 2018; Peterson et al., 2006). It has been also shown that practicing gymnastics in childhood and adolescence enhances the development and functioning of the postural control system (Busquets et al., 2018; Glomer et al., 1997; Kioumourtzoglou et al., 1997). The gymnastic experience may lead to decreased dependence on visual inputs in postural control of standing on an unstable surface (Glomer et al., 1997). It may also stimulate the development of proprioceptive reweighting processes in children (Busquets et al., 2018). However, not all methods used to investigate postural control turned out to be sensitive enough to demonstrate the superior abilities of this population. For example, the assessment of traditional center of pressure (COP) measures acquired during quiet standing did not indicate superior postural steadiness in young adolescent gymnasts compared to that of their non-athlete peers (Garcia et al., 2011; Hernández Suárez et al., 2013; Opala-Berdzik et al., 2018). This suggests that quiet standing may be a task which is too easy for the gymnasts to make use of their trained abilities. Yet, it is also possible that analyses restricted to traditional COP measures may be insufficient to distinguish between-group differences in quiet standing.

To the authors' best knowledge, the characteristics of functional stability based on the limits of stability (LOS) test have not been investigated in gymnasts regardless of their age. The LOS are defined as volitional control of the maximal center of gravity displacement in any direction without losing balance or taking a step. The LOS can be reliably assessed while the subject stands on the force plate and voluntarily assumes a maximal leaning position in a certain direction. This way the COP excursions towards the anatomically defined limits of the base of support are recorded (Alsalaheen et al., 2015; Juras et al., 2008). The LOS test, to a certain extent, imitates functional daily living tasks. In comparison to quiet standing, the transition of the body from a vertical to a maximal leaning position is associated with increased external gravitational torque and muscle engagement ( $\mathrm{Ku}$ et al., 2016). It can be assumed that the assessment of this more challenging task would be useful to demonstrate different postural control characteristics in young gymnasts. The LOS were found to be positively correlated with lower extremity muscle strength (Binda et al., 2003; Riach and Starkes, 1993), therefore, it is worth investigating whether gymnastics training results in increased stability limits in adolescence.

The investigation of the gymnasts' functional stability based on traditional COP measures can be expanded by stabilogram decomposition into rambling (RM) and trembling (TR) components to enable the detection of even subtle differences in postural control characteristics. The RM component has been defined as an instantaneous equilibrium reference point and the TR component is a fluctuation of the COP around the reference point (Zatsiorsky and Duarte, 1999, 2000). It has been proposed that the RM component reflects the processes of the CNS that are controlled by the supraspinal centers (i.e., the brain), while the TR component reflects the peripheral mechanisms of the postural control system, such as spinal reflexes and/or passive mechanical properties of the muscles, ligaments and joints (Shin at al., 2019). The ramblingtrembling (RM-TR) measures have been considered reliable (Słomka et al., 2013).

In healthy athlete and non-athlete children and young adolescents, decreased COP measures have been interpreted as better postural stability (Garcia et al., 2011). In turn, according to an assessment of RM-TR measures in healthy untrained children, adolescents, and adults, lower RM values may indicate a better estimation of the overall body position and velocity, and reduced TR may suggest a smaller discrepancy between action planning and performance (i.e., reduced noise in the postural control system) (Ferronato and Barela, 2011).

The aim of this study was to determine whether young adolescent female artistic gymnasts would demonstrate better forward functional stability compared to that of their ageand sex-matched non-athlete counterparts. The authors hypothesized that the gymnasts' superior postural control would be observed during the transition to and standing in the maximal forward leaning position.

\section{Methods}

This study is a part of the project regarding the assessment of postural stability in 
young adolescent gymnasts which was conducted following the approval of the Institutional Bioethics Committee.

\section{Participants}

Twenty-two 10- to 13-year-old healthy females (ten artistic gymnasts and twelve nonathletes) participated in the study. The gymnasts were recruited from training centers and the nonathletes were recruited from an elementary school in Upper Silesia, Poland. The inclusion criteria for the gymnasts involved being at the national performance level and practicing for about 12 hours per week. The inclusion criteria for the nonathlete females were no experience in practicing sports and participation only in general physical education classes at the elementary school (3 hours per week). Exclusion criteria were uncorrectable vision disorders, obesity, and any neurological or musculoskeletal abnormalities that could affect postural stability. The characteristics of the young adolescent females are given in Table 1 . The aim of the study and experimental procedures were explained to all study participants and their legal guardians, and a written informed consent was obtained from the participants and guardians.

\section{Procedures}

Prior to the balance assessment, the measurements of the subjects' body height and mass were taken. Individual body mass index (BMI) percentile rankings were calculated according to the international BMI cut-off points for female children established by the International Obesity Task Force (Table 1).

Functional stability was assessed with the use of the LOS test (Juras et al., 2008) performed on a stable force plate (AMTI AccuGait, Watertown, MA, USA). Participants were instructed to stand barefoot on the force plate with their feet approximately hip-width apart and arms at their sides. In order to keep the same position of the feet on the force plate between trials, a $1 \mathrm{~cm}$ thick line was drawn $10 \mathrm{~cm}$ away from the front edge of the plate and subjects were asked to place their big toes so that they were tangential to the drawn line. Subjects' gaze was focused on a target set approximately $3 \mathrm{~m}$ away in front of their eye level. The test lasted 30s and it was divided into three distinct phases: $1^{\text {st }}$ phase quiet standing (10s), $2^{\text {nd }}$ phase - pendulum like shift to maximal forward leaning position (after an acoustic signal), and $3^{\text {rd }}$ phase - standing in the maximal forward leaning position. The subjects were instructed to keep their heels in contact with the plate throughout the duration of the trial and perform the leaning task only by dorsiflexing their ankle joints. They were also instructed to make the shift from quiet standing to maximal forward leaning at their own pace. Before actual measurement, the subjects performed one familiarization trial after which the LOS test was repeated twice. Between trials, the subjects were asked to get off the platform and rest for $2 \mathrm{~min}$ to avoid possible fatigue effects (Juras et al., 2008).

The forces (Fx, Fy, Fz) and moments (Mx, $\mathrm{My}, \mathrm{Mz}$ ) were registered at a $100 \mathrm{~Hz}$ sampling frequency. The raw data were processed offline with the $4^{\text {th }}$ order low pass Butterworth filter at a $7-\mathrm{Hz}$ cut-off frequency using Matlab r2017b software (Mathworks Inc., Natick, MA, USA). For the $1^{\text {st }}$ and $3^{\text {rd }}$ phases of the LOS test, the root mean squares (rms) and mean velocities (v) of COP and RM-TR in the anterior-posterior (AP) and medial-lateral (ML) directions were calculated. The rambling and trembling stabilogram decomposition was conducted according to the method established by Zatsiorsky and Duarte $(1999 ; 2000)$. The moments in which the horizontal forces applied in the force plate equaled zero were identified and recorded for both anterior-posterior and medial-lateral directions. These moments corresponded to instant equilibrium points and they were identified in the COP trajectories. A cubic spline function was used to calculate the instant equilibrium point trajectory which is considered a rambling trajectory, representing the change from one equilibrium point to another. The trembling trajectory was calculated by subtracting the approximated rambling trajectory from the corresponding COP trajectory (Zatsiorsky and Duarte, 1999; 2000) (Figure 1). Additionally, maximal forward COP excursion (the subject's anterior stability limits) was established as the distance between mean COP positions in the $1^{\text {st }}$ and $3^{\text {rd }}$ phases. All variables were computed on the basis of the means of the two trials (Juras et al., 2008).

\section{Statistical Analysis}

Statistical analysis was performed on the subjects' demographic characteristics and LOS variables. The Mann-Whitney $U$ test was used to 
compare the measures between the artistic gymnasts and non-athletes. Because of significant between-group differences in body height (Table 1), LOS variables normalized to the subject's height were compared in order to eliminate possible differences in LOS test performance attributable to this factor (Hrysomallis, 2011). The level of significance was set at $p<0.05$. The analyses were performed using Statistica v.13 software (StatSoft Inc., Tulsa, OK, USA).

\section{Table 1.}

Basic characteristics of artistic gymnasts and non-athletes

\begin{tabular}{lcc}
\hline & Artistic gymnasts $(\mathrm{n}=10)$ & Non-athletes $(\mathrm{n}=12)$ \\
\hline Age (years) & $11.1 \pm 1.29 ; 10-13$ & $10.8 \pm 0.72 ; 10-12$ \\
Body height $(\mathrm{cm})^{*}$ & $139.8 \pm 5.85 ; 132-150$ & $147.7 \pm 6.46 ; 136-155$ \\
Body mass $(\mathrm{kg})^{* *}$ & $29.6 \pm 3.60 ; 24-35.5$ & $36.5 \pm 4.46 ; 29-41.5$ \\
BMI percentile*** & $14.8 \pm 10.16 ; 3-30$ & $37.6 \pm 21.8 ; 12-84$ \\
\hline Gymnastics experience (years) & $4.8 \pm 0.29 ; 4-6$ & 0 \\
$\begin{array}{l}\text { Training frequency } \\
\text { (hours/week) }\end{array}$ & $12.5 \pm 0 ; 12.5$ & 0 \\
\hline
\end{tabular}

Data are presented as means \pm standard deviations; ranges. BMI - Body mass index. Mann-Whitney U test: ${ }^{*} p=0.007,{ }^{* *} p=0.002,{ }^{* *} p=0.002$.

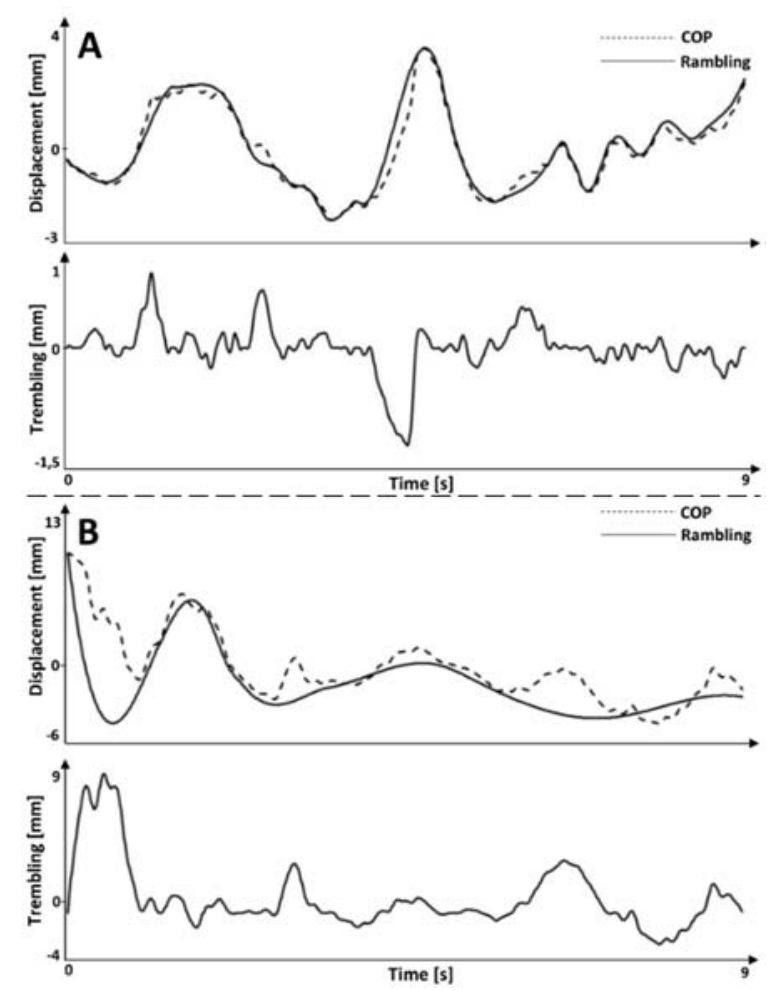

Figure 1.

Center of pressure and rambling and trembling time series of an exemplary subject for $A$ ) the $1^{\text {st }}$ phase (quiet standing) and $B$ ) the $3^{\text {rd }}$ phase (standing in maximal forward leaning position) of the LOS test. 
LOS Phase 1
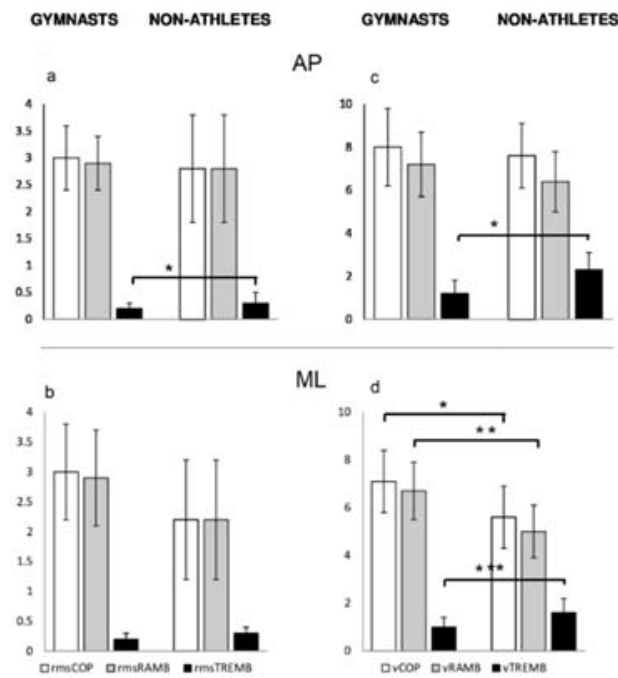

ML

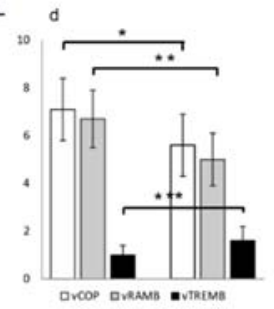

Figure 2.

First phase (10-s quiet standing) of the limits of stability (LOS) test in 10- to 13-year-old female artistic gymnasts $(n=10)$ and non-athletes $(n=12)$. Normalized to body height data are shown as means and standard deviations; $a, b$ : root mean squares (rms) and $c$, $d$ : mean velocities $(v)$ of center of foot pressure $(C O P)$, rambling $(R A M B)$ and trembling $(T R E M B)$ in the anterior-posterior $(A P)$ and medial-lateral

$(M L)$ directions. Between-group significant differences in a: ${ }^{*} p=0.02 ; c:{ }^{*} p=0.003 ; d:{ }^{*} p=0.02$, ${ }^{* *} p=0.002,{ }^{* * *} p=0.02 ;$ Mann-Whitney U tests.

LOS Phase 3

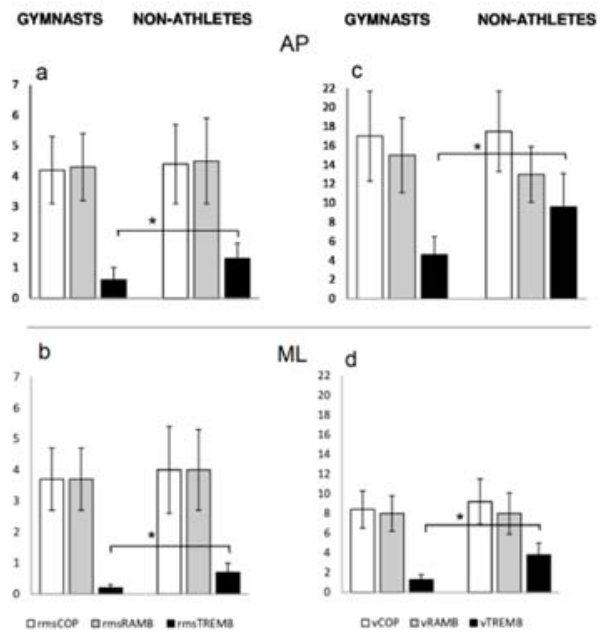

Figure 3.

Third phase (standing in maximal forward leaning position) of the LOS (limits of stability) test in 10- to 13-year-old female artistic gymnasts $(n=10)$ and non-athletes $(n=12)$.

Normalized to body height data are shown as means and standard deviations; $a$, $b$ : root mean squares (rms) and $c$, d: mean velocities $(v)$ of center of foot pressure (COP), rambling (RAMB) and trembling $(T R E M B)$ in the anterior-posterior $(A P)$ and medial-lateral $(M L)$ directions. Between-group significant differences in $a:{ }^{*} p=0.0006 ; b:{ }^{*} p=0.000003 ; c:{ }^{*} p=0.0003 ; d:{ }^{*} p=0.000006 ;$ Mann-Whitney U tests. 


\section{Results}

The results of the between-group demographic data comparison are shown in Table 1.

\section{$1^{\text {st }}$ phase of the LOS test (quiet standing)}

In the $1^{\text {st }}$ phase of the LOS test (10-s quiet standing), the Mann-Whitney U comparison showed non-significant differences in rms and mean velocities of COP and RM in the AP plane between young adolescent female artistic gymnasts and their non-athlete female peers ( $p>$ $0.05)$. However, the rms and mean velocities of TR were significantly lower in the gymnasts $(U=24$, $\mathrm{p}=0.02$ and $\mathrm{U}=17, \mathrm{p}=0.003$, respectively; Figure $2)$. In the ML plane, there were no between-group differences in rms values ( $p>0.05)$. In turn, mean velocities of the COP and RM were higher in the gymnasts compared to those of the non-athletes $(\mathrm{U}=25, \mathrm{p}=0.02$ and $\mathrm{U}=15, \mathrm{p}=0.002$, respectively), and mean velocity of TR was lower in the gymnasts $(U=25, p=0.02$; Figure 2).

$3^{\text {rd }}$ phase of the LOS test (standing in maximal forward leaning position)

The Mann-Whitney $U$ analysis for the $3^{\text {rd }}$ phase of the LOS test (standing in the maximal forward leaning position) demonstrated that in both $\mathrm{AP}$ and ML planes, the rms and mean velocities of COP and RM were not significantly different between the artistic gymnasts and nonathletes ( $p>0.05)$. Concomitantly, in both planes, the rms and mean velocities of TR were significantly lower in the gymnasts (in AP plane: $\mathrm{U}=11, \mathrm{p}=0.0006$ and $\mathrm{U}=9, \mathrm{p}=0.0003$, respectively; in ML plane: $\mathrm{U}=0, \mathrm{p}=0.000003$ and $\mathrm{U}=1, \mathrm{p}=0.000006$, respectively; Figure 3).

Anterior stability limit

The mean \pm SD of the maximal forward COP excursion (anterior stability limit) was $55.5 \pm$ 8.6 in the gymnasts and $59.7 \pm 10.1$ in the nonathletes. The statistical analysis indicated that the between-group differences concerning this measure were not significant $(p=0.28)$.

\section{Discussion}

In this study, the aim was to compare functional postural stability between 10- to 13year-old national level female artistic gymnasts and their non-athlete female peers. The authors hypothesized that gymnasts would demonstrate better postural stability during the transition to maximal forward leaning as well as standing in the maximal forward leaning position. However, the most clear picture of superior postural control in gymnasts was observed during the maintenance of static stability at their stability limit. The task of standing in the maximal forward leaning position was characterized by significantly lower values for all trembling variables.

As quiet standing and standing in maximal forward leaning phases were described by the same measures, they are discussed as first. Then, the discussion on the functional transition to the forward leaning position follows.

As expected, the present comparison of traditional COP measures acquired during quiet standing (first $10 \mathrm{~s}$ of the test) mostly indicates that the gymnasts' postural control was similar to that of non-athletes. In addition, the comparison of most rambling variables suggests that gymnastics training had no influence on the supraspinal control of body dynamics in simple quiet standing. This suggests that analysis of the simple quiet standing task may not be sufficient to distinguish among postural control characteristics between young adolescent gymnasts and nonathletes. These findings are in agreement with the literature regarding static and dynamic balance characteristics in gymnasts. Based on the review, it has been suggested that gymnasts demonstrate superior postural control under conditions which are more challenging than quiet standing (Hrysomallis, 2011). When quiet standing postural sway was compared between children practicing gymnastics and their untrained peers in different age groups, superior postural steadiness was observed in 5- to 7-year-old, but it was not shown in the older group of 9- to 11-year-old gymnasts (Garcia et al., 2011). The results of the present study regarding 10-s quiet standing also confirm those obtained in the authors' previous research in which directional subcomponents of COP velocity acquired during 60-s quiet standing were compared between young adolescent acrobatic gymnasts and non-athletes. Interestingly, the agreement concerns a puzzling result of faster medial-lateral COP excursions in the gymnasts as well (Opala-Berdzik et al., 2018). Similarly, the higher COP velocity during quiet standing was noted in young adolescent rhythmic gymnasts (Hernández Suárez et al., 2013). In the present 
study, artistic gymnasts also demonstrated faster medial-lateral rambling displacements. The mechanisms underlying the tendency to faster quiet standing sway in adolescent gymnasts are unknown and require further investigation; however, they may be related to motor abundance, which allows athletes to perform very challenging motor tasks and overcome larger perturbations (Latash, 2012). On the other hand, the present study suggests that during 10-s quiet standing which initiated the LOS test gymnasts presented slower anterior-posterior and mediallateral trembling excursions as well as lower values for the anterior-posterior rms of trembling compared to those of non-athletes. This indicates that gymnastics training may have led to reduced noise in the postural control of quiet standing (Ferronato and Barela, 2011). A recent study on healthy subjects has shown a positive correlation between the trembling component of quiet standing postural sway and lower extremity muscle stiffness (Shin at al., 2019), therefore, it can be assumed that the reduction of the trembling component in gymnasts might have been related to better contractile properties of their lower extremity muscles.

In the present study, the last phase of the LOS test required standing in the maximal forward leaning position for about $15 \mathrm{~s}$. The comparison of this task clearly showed similarities and differences in functional stability between the young adolescent artistic gymnasts and their untrained peers. No between-group differences were observed in COP and RM excursions, which may suggest similar supraspinal control of the body position during standing with the COP moved to the anterior stability limit. However, during this task TR displacements were smaller and slower in gymnasts (in both the AP and ML directions). This may imply that due to gymnastics experience, the inherent postural noise was mitigated by superior control processes at the spinal level possibly related to better lower extremity muscle contractile properties (Shin at al., 2019).

The present study also suggests that analyses restricted to the traditional COP variables of the LOS test were not sufficient to expose postural stability characteristics specific to young adolescent gymnasts and their non-athlete peers. The RM-TR stabilogram decomposition allowed the determination of differences in functional stability between gymnasts and untrained study participants.

In this study, it was hypothesized that artistic gymnastics training could result in increased anterior stability limit in young adolescent females. This assumption was based on previous findings which indicated a positive correlation between anterior-posterior LOS and lower extremity muscle strength (Binda et al., 2003; Riach and Starkes, 1993). However, the present results suggest that in 10- to 13-year-old healthy females, the gymnastics experience did not result in an increased range of maximal voluntary forward leaning. It is possible that the anterior stability limit was mainly influenced by the anthropometric characteristics of the young adolescent females. It has been recently documented that when the maximal forward leaning position is maintained with heels in contact with the ground, the forward anatomical stability limit is located at the level of first metatarsophalangeal joints (Słomka et al., 2020). In turn, according to the research conducted in seventy children aged 4-14 years, the most important factors determining their anteriorposterior LOS were body height and foot length. These two factors were also highly correlated with each other (Riach and Starkes, 1993). In the present study, the anterior stability limits were normalized to the participants' body height; however, this procedure could have been insufficient to eliminate the influence of foot length/body height on this measure, especially because gymnasts were notably shorter than nonathletes (by $8 \mathrm{~cm}$ on average). However, a limitation of the present study is the lack of a foot measurement, which would allow the data to be normalized according to the participant's foot length. Adequate foot measurements would also enable calculation of forward functional stability indicator for the study participants (Słomka et al., 2020). Because of the relatively small sample size of this study, the results should be interpreted with caution.

In conclusion, during the most challenging phase of the LOS test comprised of standing in the maximal forward leaning position, the differences in postural stability characteristics between young adolescent female artistic 
gymnasts and their non-athlete female peers were most pronounced. In that phase, trembling displacements were slower and smaller in gymnasts indicating lesser noise in their postural control system. The LOS test with the use of rambling and trembling stabilogram decomposition provided valuable information regarding the influence of artistic gymnastics training on the young adolescents' postural control.

\section{References}

Alsalaheen B, Haines J, Yorke A, Broglio SP. Reliability and construct validity of limits of stability test in adolescents using a portable forceplate system. Arch Phys Med Rehabil, 2015; 96(12): 2194-2200.

Barela JA, Jeka JJ, Clark JE. Postural control in children: Coupling to dynamic somatosensory information. Exp Brain Res, 2003; 150: 434-442.

Binda SM, Culham EG, Brouwer B. Balance, muscle strength, and fear of falling in older adults. Exp Aging Res, 2003; 29: 205-219.

Busquets A, Aranda-Garcia S, Ferrer-Uris B, Marina M, Angulo-Barroso R. Age and gymnastic experience effects on sensory reweighting processes during quiet stand. Gait Posture, 2018; 63: 177-183.

Ferronato PAM, Barela JA, Age-related changes in postural control: rambling and trembling trajectories. Motor Control, 2011; 15: 481-493.

Figura F, Cama G, Capranica L, Guidetti L, Pulejo C. Assessment of static balance in children. J Sports Med Phys Fitness, 1991; 31(2): 235-242.

Garcia C, Barela JA, Viana AR, Barela AM. Influence of gymnastics training on the development of postural control. Neurosci Lett, 2011; 492: 29-32.

Hernández Suárez M, Guimeraes-Ribeiro D, Hernández Rodriguez JE, Rodríguez-Ruíz D, García-Manso JM. The effect of early systematic gymnastics training on postural control. Abstracts from the 3rd European College of Sports and Exercise Physicians conference on 25-27 April 2013. Br J Sports Med, 2013; 47: 31.

Golomer E, Dupui P, Monod H. The effects of maturation on self-induced dynamic body sway frequencies of girls performing acrobatics or classical dance. Eur J Appl Physiol, 1997; 76: 140-144.

Goulème N, Debue M, Spruyt K, Vanderveken C, De Siati RD, Ortega-Solis J, Petrossi J, Wiener-Vacher S, Bucci MP, Ionescu E, Thai-Van H, Deggouj N. Changes of spatial and temporal characteristics of dynamic postural control in children with typical neurodevelopment with age: Results of a multicenter pediatric study. Int J Pediatr Otorhinolaryngol, 2018; 113: 272-280.

Hrysomallis C. Balance ability and athletic performance. Sport Med, 2011; 41(3): 221-232.

Ivanenko Y, Gurfinkel VS. Human Postural Control. Front Neurosci, 2018; 12: 171.

Juras G, Słomka K, Fredyk A, Sobota G, Bacik B. Evaluation of the limits of stability (LOS) balance test. J Hum Kinet, 2008; 19: 39-52.

Kioumourtzoglou E, Derri V, Mertzanidou O, Tzetzis G. Experience with perceptual and motor skills in rhythmic gymnastics. Percept Mot Skills, 1997; 84: 1363-1372.

Ku PX, Abu Osman NA, Wan Abas WAB. The limits of stability and muscle activity in middle-aged adults during static and dynamic stance. J Biomech, 2016; 49: 3943-3948.

Latash ML. The bliss (not the problem) of motor abundance (not redundancy). Exp Brain Res, 2012 ; 217 : 1-5.

Newell KM, Slobounov SM, Slobounova BS, Molenaar PCM. Short-term non-stationarity and the development of postural control. Gait Posture, 1997; 6: 56-62.

Opala-Berdzik A, Głowacka M, Wilusz K, Kołacz P, Szydło K, Juras G. Quiet standing postural sway of 10to 13-year-old, national-level, female acrobatic gymnasts. Acta Bioeng Biomech, 2018; 20(2): 117-123.

Peterson ML, Christou E, Rosengren KS. Children achieve adult-like sensory integration during stance at 12years-old. Gait Posture, 2006; 23: 455-463.

Riach CL, Starkes JL. Stability limits of quiet standing postural control in children and adults. Gait Posture, 1993; 1: 105-111. 
Shin S, Milosevic M, Chung C-m, Lee Y. Contractile properties of superficial skeletal muscle affect postural control in healthy young adults: A test of the rambling and trembling hypothesis. PLoS ONE, 2019; 14(10): e0223850.

Słomka K, Juras G, Sobota G, Bacik B. The reliability of a rambling-trembling analysis of center of pressure measures. Gait Posture, 2013; 37: 210-213.

Słomka KJ, Michalska J, Marszałek W, Bacik B, Juras G. Forward functional stability indicator (FFSI) as a reliable measure of limits of stability. MethodsX, 2020; 7: 100756.

Usui N, Maekawa K, Hirasawa Y. Development of the upright postural sway of children. Develop Med Child Neurol, 1995; 37: 985-996.

Zatsiorsky VM, Duarte M. Instant equilibrium point and its migration in standing tasks: rambling and trembling components of the stabilogram. Motor Control, 1999; 3: 28-38.

Zatsiorsky VM, Duarte M. Rambling and trembling in quiet standing. Motor Control, 2000; 4: 185-200.

\section{Corresponding author:}

\section{Agnieszka Opala-Berdzik}

Institute of Physiotherapy and Health Sciences,

Department of Physiotherapy in Internal Diseases,

The Jerzy Kukuczka Academy of Physical Education in Katowice,

Ul. Mikołowska 72; 40-065 Katowice, Poland

Tel. +48 322075301

E-mail: a.opala-berdzik@awf.katowice.pl 